\title{
ROS-triggered AMPK de-SUMOylation promotes Warburg effect in hepatocellular carcinoma cells
}

\author{
Shutian Zhang
}

The authors have withdrawn this manuscriot as they no longer stand by the conclusions.

As found in previous literature review, the regulation effect and mechanism of reactive oxygen species (ROS) on the metabolism and proliferation of tumor cells are very complex. The preliminary results in the preprint may be just one front of "ROS-AMPKtumor cell metabolism". After further experiments, the effects of different ROS concentrations on the metabolism of tumor cells as the cell passage increased were not completely consistent with the results shared in the preprint, so the author decided to withdraw the data in this preprint. The author do not wish this version to be cited as reference for this study. Subsequent investigators should carefully evaluate the consistency of the role of ROS stimulation in cell studies in vitro. 